\title{
The comparison of rDNA spacer regions of Nosema ceranae isolates from different hosts and locations
}

\author{
Wei-Fone Huang ${ }^{\text {a }}$, Michel Bocquet ${ }^{\mathrm{b}}$, Ker-Chang Lee ${ }^{\mathrm{a}}$, I-Hsin Sung ${ }^{\mathrm{c}}$, Jing-Hao Jiang ${ }^{\mathrm{a}}$, \\ Yue-Wen Chen ${ }^{\mathrm{d}}$, Chung-Hsiung Wang ${ }^{\mathrm{a}, *}$ \\ a Department of Entomology, National Taiwan University, No. 1, Sec. 4, Roosevelt Rd., 106 Taipei, Taiwan \\ ${ }^{\mathrm{b}}$ Individual Researcher, BP 22, 74371 PRINGY Cedex, France \\ ${ }^{c}$ Tainan District Agricultural Research and Extension Station, Council of Agriculture, Executive Yuan, 712 Tainan, Taiwan \\ ${ }^{\mathrm{d}}$ Department of Animal Science, National I-Lan University, I-Lan 260, Taiwan
}

Received 12 October 2006; accepted 9 July 2007

Available online 18 July 2007

\begin{abstract}
Nosema ceranae is a common microsporidian pathogen, one of two Nosema species that cause "nosema disease" in honeybees, Apis cerana and Apis mellifera. Samples of $N$. ceranae rDNA from isolates collected in different locations were sequenced and one 5S rRNA was found to be upstream of SSUrRNA. The rDNA arrangement, 5'-5S rRNA-IGS-SSUrRNA-ITS-LSUrRNA-3', was found in all isolates. In order to better understand the distribution relationship between $N$. ceranae isolates from $A$. cerana and $A$. mellifera, their rRNA spacer regions were also sequenced for analysis. Results showed that there are no significant differences between the IGS sequences of the isolates and no difference in the ITS sequence with the exception of one transition found in an isolate from Martinique. These isolates showed consistency in the IGS phylogenic analysis suggesting that no transmission barrier exists between $A$. mellifera and A. cerana and there is no difference between isolates from geography separated areas.
\end{abstract}

(C) 2007 Elsevier Inc. All rights reserved.

Keywords: Nosema ceranae; Nosema disease; rDNA; IGS; ITS; France; Martinique; Spain; Taiwan

\section{Introduction}

Nosema ceranae is one of two microsporidian pathogens causing "nosema diseases" in honeybees, Apis cerana and Apis mellifera. Nosema disease is not uncommon in temperate climates and could be devastating to honey production (Fries and Ekbom, 1984). Nosema apis was first observed by Zander (1909) in A. mellifera and can now be found worldwide (Matheson, 1996) in A. mellifera and in A. cerana (Singh, 1975). N. ceranae was first recovered from A. cerana by Fries et al. (1996). The authors compared the morphological and ultrastructure characters between $N$. apis and $N$. ceranae and found that the

\footnotetext{
rDNA of Nosema ceranae isolates from different hosts and locations.

* Corresponding author. Fax: +886 227364329.

E-mail address: wangch@ntu.edu.tw (C.-H. Wang).
}

SSUrRNA sequences differed between the two species. The spores of $N$. apis and $N$. ceranae are also somewhat different. The spore of $N$. ceranae is shorter on average and ultrastructural studies revealed fewer polar filament coils in $N$. ceranae, 20-23 compared to 30-44 in N. apis (Fries, 1989). Lower identity of SSUrRNA sequences from $N$. apis and N. ceranae has been reported compared to the sequences from other Nosema species utilizing the same host (Huang et al., 2007). Recently, N. ceranae was found in cultivated A. mellifera colonies (Huang et al., 2005; Higes et al., 2006; Huang et al., 2007). It is not known whether $N$. ceranae is a new, emerging honeybee parasite or is a naturally occurring species that had been overlooked due to its morphological similarity to $N$. apis, but its success in two closely related hosts and close molecular relationship suggest an historic presence in at least one species of honeybees. 
New findings enable us to examine the details of $N$. ceranae's rDNA arrangement. The SSUrRNA gene of $N$. cerana was sequenced to confirm the transcript initiation site and the reversed orientation of $5 \mathrm{~S}$ rRNA in $N$. ceranae was found to be upstream of SSUrRNA. This gene arrangement seems to be unique to $N$. ceranae because no such arrangement was found in $N$. apis, $N$. bombycis and other closely related species (Huang et al., 2004; Tsai et al., 2005; Ku et al., 2007).

To further study $N$. ceranae distribution, we obtained the $N$. ceranae isolates from $A$. mellifera collected from several different geographic locations and $A$. cerana in Taiwan. We report here the intergenic spacer region, IGS (between 5SrRNA and SSUrRNA), and the ITS regions of all isolates. We use comparisons and analyses of these sequences to establish the relationship among these $N$. ceranae isolates.

\section{Materials and methods}

\subsection{N. ceranae and N. apis isolates}

Samples of $N$. ceranae and $N$. apis were recovered from honeybees collected from Taiwan and from several overseas sites. All microsporidium samples from Taiwan were identified as $N$. ceranae and were separated into three groups: those recovered from $A$. mellifera colonies in Taipei (Group 1) (Huang et al., 2007); Group 2 recovered from $A$. cerana in an apiary in I-Lan, Taiwan; and Group 3 recovered from a wild $A$. cerana colony collected at an altitude of $800 \mathrm{~m}$ in a southern Taiwan mountain area. The overseas samples included both $N$. ceranae and $N$. apis. Spain's isolates of N. ceranae and N. apis from A. mellifera were gifts from Dr. Mariano Higes. The $N$. ceranae samples from Martinique Island and France were isolated by Michel Bocquet from infected A. mellifera colonies. All isolates were collected in the years 2004-2006 (Table 1).

\subsection{DNA purification}

The DNA of the isolates from Spain was purified according to a method reported by Higes et al. in 2006 .
The DNA of Taiwan Group 1 was previously isolated (Huang et al., 2007). The remaining DNA samples were each isolated from one ventriculus of $A$. cerana or A. mellifera. The ventriculus was ground in TE buffer $(0.1 \mathrm{M}$ Tris, $0.01 \mathrm{M}$ EDTA, $\mathrm{pH}$ 9.0) followed by Proteinase K treatment. The phenol/chloroform extraction method was then used to remove most protein and was followed by alcohol precipitation. The DNA pellets were dissolved in $\mathrm{ddH}_{2} \mathrm{O}$ and stored at $-20^{\circ} \mathrm{C}$.

\subsection{PCR amplification strategy}

The upstream region of SSUrRNA was sequenced by SSP-PCR (single-specific-primer PCR; Shyamala and Ames, 1989). A specific primer 5SF (ACA AAA ACT ACA GCA CCT AGC) was then designed based on the 5S rRNA sequence. The SSUrRNA gene of each sample was sequenced as described previously (Huang et al., 2007). Fig. 1 depicts the primer annealing sites for spacer region amplification. The IGS region of the ribosomal gene repeat unit was amplified through the primer set 5SF and SSU5r (ACG TCA GGC AGA ATC AAC). The ITS region was amplified using the primer set, S1129f (TGA ATG TGT CCC TGT TCT TTG) and L1328r (GGT ATC CTA TTG ATC CCA TGT G). The PCR mixture was prepared according to the manufacturer's instructions (HiFi DNA polymerase, Yeastern Biotech) and amplifications were performed in an AG-9600 Thermal Station (Biotronics Corp.). A $10 \mu \mathrm{l}$ aliquot from each reaction was run on a $1.0 \%$ agarose gel to visualize the PCR products. These products were then eluted using a DNA Clean/Extraction Kit (Genemark) and cloned into a T\&A cloning vector (Real Biotech Corp.). The inserted DNA fragments were sequenced on an automated DNA Sequencer (DNA Sequencer 377, Applied Biosystems).

\subsection{Phylogenetic analyses}

Because the intra-isolate variations of the isolate from Spain and two isolates (wild A. cerana and A. mellifera) from Taiwan are high (more than 3\%), two IGS sequences from these isolates were added to the phylogenetic analysis.

Table 1

Spacer region sequences of Nosema ceranae and Nosema apis isolates

\begin{tabular}{|c|c|c|c|c|c|c|}
\hline \multirow[t]{2}{*}{ Species } & \multirow[t]{2}{*}{ Isolated region } & \multirow[t]{2}{*}{ Host } & \multicolumn{3}{|c|}{ Sequences } & \multirow[t]{2}{*}{ Reference } \\
\hline & & & ITS $^{\mathrm{a}}$ & IGS & IGS GenBank Accession No. & \\
\hline \multirow[t]{5}{*}{ N. ceranae } & Taiwan, Asia & A. mellifera & $39 \mathrm{bp}$ & $606 \mathrm{bp}$ & EF091879 & \\
\hline & Taiwan, Asia & Wild A. cerana & $39 \mathrm{bp}$ & $614 \mathrm{bp}$ & EF091884 & \\
\hline & France, Europe & A. mellifera & $39 \mathrm{bp}$ & $606 \mathrm{bp}$ & EF091881 & \\
\hline & Spain, Europe & A. mellifera & $39 \mathrm{bp}$ & $607 \mathrm{bp}$ & EF091880 & \\
\hline & Martinique, America & A. mellifera & $39 \mathrm{bp}$ & $606 \mathrm{bp}$ & EF091882 & \\
\hline Nosema sp. & France, Europe & A. mellifera & $28 \mathrm{bp}$ & N/A & N/A & \\
\hline
\end{tabular}

\footnotetext{
${ }^{a}$ The ITS sequences alignments are shown in Fig. 3.
} 


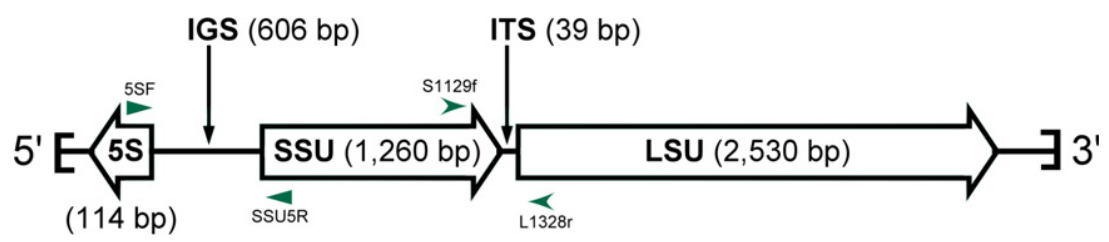

Fig. 1. Schematic diagram of the Nosema ceranae rRNA genes. The rRNA domains are indicated by hollow arrows and the direction of arrow indicates the direction of transcription. The small arrowheads represent primers used to amplify the IGS and ITS regions.

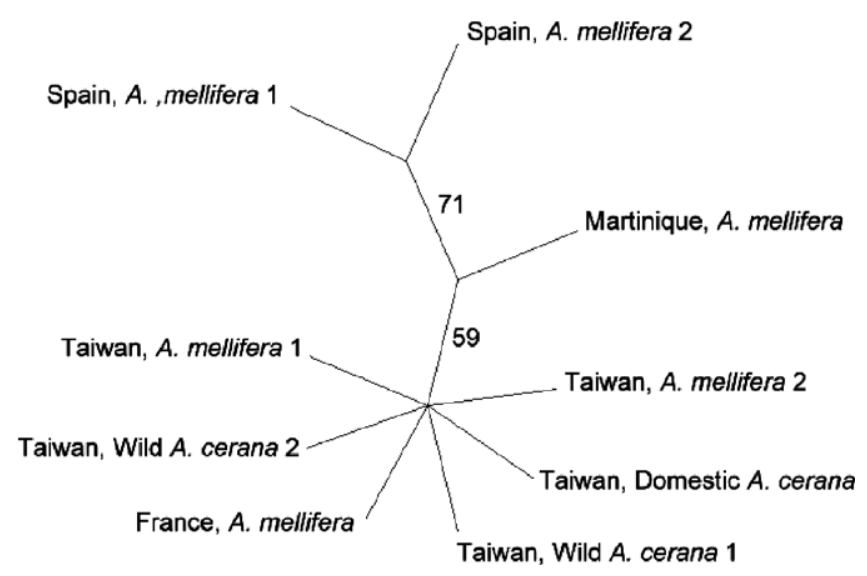

Fig. 2. Unrooted phylogenetic tree based on IGS sequences. Nine taxa were analyzed based on a maximum likelihood approach using PAUP 4.0 b10 (Swofford, 2003), run with 100 bootstrap replications. Numbers indicate branch support statistics.

One is the consensus sequence (those with number 1 on the phylogenetic tree; Fig. 2) and the other is the sequence with variation (number 2 on the phylogenetic tree). In the phylogenetic analysis, we used the consensus sequences for the IGS sequences of the samples from Martinique, France, and domestic $A$. cerana in Taiwan, because their intra-isolate variations are lower. Sequences were aligned using ClustalX (Thompson et al., 1997). Poorly aligned regions were excluded manually. Phylogenetic analyses were conducted based on maximum likelihood (ML) with PAUP 4.0 b10 (Swofford, 2003). The substitution model of the ML analysis was set according the HKY model and the variable sites of among site rate variation followed gamma distribution. It was set according to the Modeltest (Posada and Crandall, 1998) result. The rate matrix, base frequency, proportion of invariable sites, and shape parameter were estimated. Node support of ML analyses was assessed with 100 bootstrap replicates.

\section{Results}

The SSUrRNA of each of the $N$. ceranae isolates was sequenced and confirmed to be $N$. ceranae. The SSUrRNA of all $N$. ceranae isolates found to be polymorphic, including the isolates from a single ventriculus. The polymorphic sites are similar to those previous published (Huang et al., 2007). The $5^{\prime}$ region of SSUrRNA was sequenced and found to be 5'-5S rRNA-IGS-SSUrRNA-ITSLSUrRNA-3' (Fig. 1). The orientation of 5S rRNA in
$N$. ceranae is the reverse of SSUrRNA and LSUrRNA, and the length of the 5S rRNA is $114 \mathrm{bp}$. The 5S rRNA in $N$. ceranae shares $84-93 \%$ similarity with other known microsporidia. The IGS is AT rich (about $83 \%$ ) and polymorphic, and it is approximately $606 \mathrm{bp}$ long. The IGS regions of all $N$. ceranae isolate from different locations were sequenced and submitted to GenBank (accession numbers are listed in Table 1), and the similarity among isolates was $94-97 \%$. The phylogenetic tree of the IGS region (Fig. 2) included more than one sequence from some isolates in the analysis that showed the polymorphism effect. The phylogenetic tree indicates that the isolates from Spain were closely related to their counterparts from Martinique.

The ITS sequence of all isolates consists of only 39 base pairs (Table 1) and the alignment of these sequences is shown (Fig. 3). The ITS sequences were highly conserved in both $N$. ceranae and $N$. apis. Only one transition was found in the $N$. ceranae samples collected from Martinique. A similar result was found in $N$. apis, with only one transition between the ITS sequence of isolate from Spain and the ITS sequence (U97150) of the $N$. apis collected in New Zealand.

\section{Discussion}

The SSUrRNA sequences of $N$. ceranae samples collected in Spain, Martinique, and Taiwan all proved to be polymorphic, and the similarity was $98-100 \%$. However, the ITS is conserved within the Nosema species infest honeybee, and it is only $50-57 \%$ identical among them. The ITS sequence seems to be a better marker than the SSUrRNA.

The discovery of the unique configuration of the $5 \mathrm{~S}$ rRNA in $N$. ceranae was unanticipated. The SSP PCR was performed to sequence the $5^{\prime}$ uncoded region of SSUrRNA and certify the transcription initiation site. The 5S rRNA was found in the upstream sequence of SSUrRNA, and its orientation was reversed compared to SSUrRNA and LSUrRNA in the rDNA repeat unit. This reversed orientation has never been reported in other known microsporidian 5S rRNA located near the rDNA repeat unit. The microsporidian 5S rRNA is usually located downstream of the rDNA repeat unit. Moreover, rDNA head-to-tail or palindromic repeats may not explain the reverse orientation of $5 \mathrm{~S}$ rRNA, and the $5 \mathrm{~S}$ rRNA may not be located downstream of LSUrRNA. (664 bp 

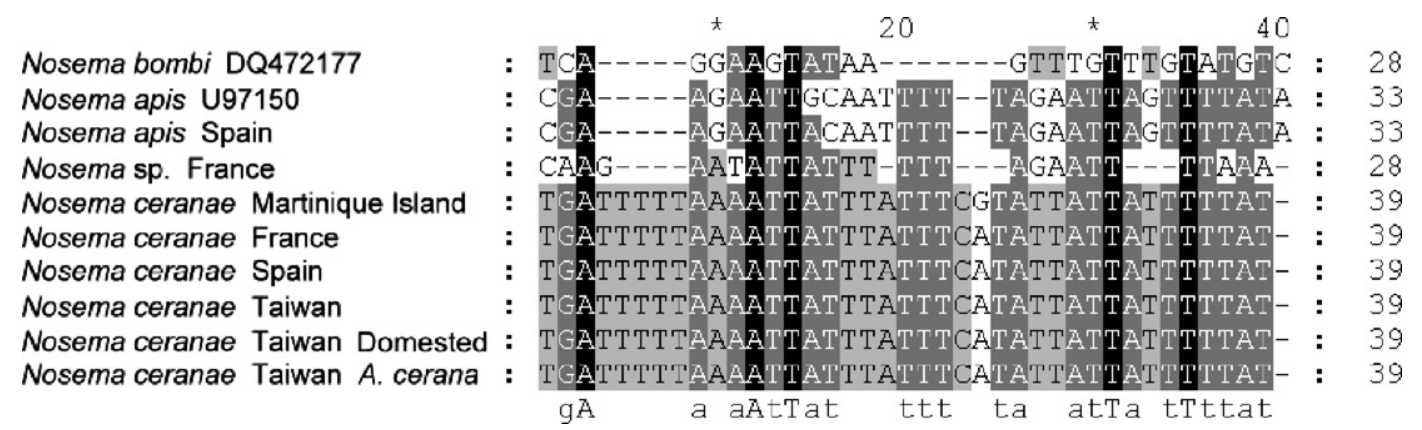

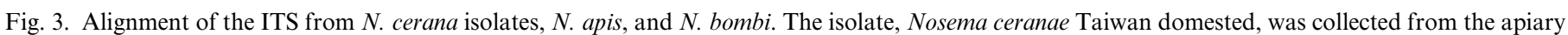

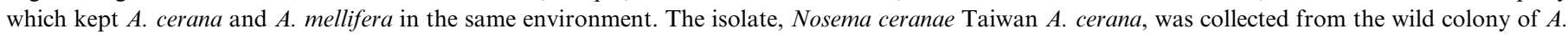
cerana. Other isolates were all collected from $A$. mellifera colonies.

downstream of the putative stop site were sequenced, Huang et al., 2007.) We speculate that the 5S rRNA of $N$. ceranae might be dispersed randomly between genes as is the 5S rRNA gene in the Encephalitozoon cuniculi genome (Katinka et al., 2001) and is fortuitously inserted upstream of an rDNA repeat. This arrangement may not exist in every rDNA repeat unit, because the IGS region seems to be more difficult to amplify through PCR. Further research will be needed to clarify this situation.

The IGS is polymorphic, and the consensus sequences of the IGS from different isolates share $94-97 \%$ similarity. The IGS is an uncoded region and more variable than the SSUrRNA of $N$. ceranae. We attempted to establish the relationship of the isolates from France, Martinique, Spain, and Taiwan via phylogenic tree analysis of the IGS sequences. However, the IGS sequences showed consistency. The isolates cannot be definitely differentiated in the analysis, except the isolates from Spain and Martinique show a close relationship. This result is unexpected as Martinique is French Caribbean Island but there may be frequent interaction among honeybee colonies from the nearby islands. The IGS sequences from $A$. cerana isolate showed little difference from those isolated from $A$. mellifera suggesting that there might be no barrier for transmission between these two hosts. However, the precise means by which $N$. ceranae is transmitted is not clear, nor is means for transmission between A. mellifera and A. cerana known. Water and food contamination and interactions among bees were considered transmission pathways between colonies for nosema disease, but $A$. mellifera and A. cerana differ in behavior and flower preference. We suggest that robbing behavior between the two species sometimes observed in apiaries could be a possible transmission pathway between A. mellifera and A. cerana.

The ratio of $N$. ceranae to $N$. apis or other microsporidia causing nosema disease may vary in different geographic locations. In Spain, the ratio of $N$. ceranae infection in colonies infected with nosema disease was reported to be as high as $90 \%$ (Higes et al., 2006). In the samples from Taiwan and Martinique, only $N$. ceranae was recovered. No co-infections were found in these samples. The SSUrRNA (GenBank Accession No. EF585399) of the unidentified microsporidium from France shares 96\% identity with $N$. ceranae and $94 \%$ identity with $N$. apis. Moreover, the ITS region was sequenced and aligned with the other ITS sequences (Fig. 3); there were noticeable differences from both $N$. apis and $N$. ceranae. The unique rDNA arrangement of $N$. ceranae was not found in this species.

The results of these studies confirmed that $N$. ceranae has already spread in many areas of the world (Klee et al., 2007). The rDNA arrangement in every $N$. ceranae isolate we tested was unique in its location and reverse arrangement. Although the IGS region of $N$. ceranae is polymorphic, the ITS region is conserved. The ITS of $N$. apis may be also quite conserved unlike the polymorphic ITS found in N. bombycis (Huang et al., 2004). The unidentified species found in France suggested that in addition to $N$. ceranae and $N$. apis, other Nosema species might be infecting A. mellifera. The rDNA sequence (including SSUrRNA and ITS) of the unidentified species is an intermediate between those of $N$. apis and $N$. ceranae, generating interesting questions about in nosema disease research.

\section{Acknowledgments}

The authors thank the Council of Agriculture, Executive Yuan, Republic of China for financially supporting this research under the Contract No. 96AS-14.3.1-BQ-B2 (8). The authors thank Dr. Eddie Chio and Dr. Leellen Solter for constructive revision of the text writing.

\section{References}

Fries, I., 1989. Observation on the development and transmission of Nosema apis Z. in the ventriculus of the honeybee. J. Apic. Res. 28, 107-117.

Fries, I., Feng, F., da Silva, A., Slemenda, S.B., Pieniazek, N.J., 1996. Nosema ceranae n. sp. (Microspora, Nosematidae), morphological and molecular characterization of a Microsporidian parasite of the Asian Honey bee Apis cerana (Hymenoptera, Apidae). Eur. J. Protistol. 32, 356-365.

Fries, I., Ekbom, G., 1984. Nosema apis, sampling techniques and honey yield. J. Apic. Res. 23, 102-105.

Gatehouse, H.S., Malone, L.A., 1998. The ribosomal RNA gene region of Nosema apis (Microspora): DNA sequence for small and large subunit rRNA genes and evidence of a large tandem repeat unit size. J. Invertebr. Pathol. 71, 97-105. 
Higes, M., Martín, R., Meana, A., 2006. Nosema ceranae, a new microsporidian parasite in honeybees in Europe. J. Invertebr. Pathol. 92, 93-95.

Huang,W.F., Jiang, J.H., Wang, C.H., 2005. Nosema ceranae infection in Apis mellifera. 38th Annual Meeting of Society for Invertebrate Pathology. Anchorage, Alaska.

Huang, W.F., Jiang, J.H., Chen, Y.W., Wang, C.H., 2007. A Nosema ceranae isolate from the honeybee Apis mellifera. Apidologie. 38, 30-37.

Huang, W.F., Tsai, S.J., Lo, C.F., Soichi, Y., Wang, C.H., 2004. The novel organization and complete sequence of the ribosomal gene of Nosema bombycis. Fungal Genet. Biol. 41, 473-481.

Katinka, M.D., Duprat, S., Cornillot, E., Metenier, G., Thomarat, F., Prensier, G., Barbe, V., Peyretaillade, E., Brottier, P., Wincker, P., Delbac, F., El Alaoui, H., Peyret, P., Saurin, W., Gouy, M., Weissenbach, J., Vivares, C.P., 2001. Genome sequence and gene compaction of the eukaryote parasite Encephalitozoon cuniculi. Nature $414,450-453$

Klee, J., Beasana, A.M., Genersch, E., Gisder, S., Nanetti, A., Tam, D.Q., Chinh, T.X., Puerta, F., Ruz, J.M., Kryger, P., Message, D., Hatjina, F., Korpela, S., Fries, I., Paxton, R.J., 2007. Wildespread dispersal of the microsporidian Nosema ceranae, an emergent pathogen of the western honey bee, Apis mellifera. J. Invertebr. Pathol. 96, 1-10.
Ku, C.C., Wang, C.Y., Tsai, Y.C., Tzeng, C.C., Wang, C.H., 2007. Phylogenetic analysis between two putative Nosema isolates from Cruciferous Lepidopteran pests in Taiwan. J. Invertebr. Pathol. 95, 71-76.

Posada, D., Crandall, K.A., 1998. Modeltest: testing the model of DNA substitution. Bioinformatics 14, 817-818.

Matheson, A., 1996. World bee health update 1996. Bee World 77, 45-51.

Shyamala, H., Ames, G.F., 1989. Genome walking by single-specificprimer polymerase chain reaction: SSP-PCR. Gene 84, 1-8.

Singh, Y., 1975. Nosema in Indian honey bee (Apis cerana indica). Am. Bee J. 115, 59.

Swofford, D.L., 2003. PAUP*, phylogenetic analysis using parasimony (* and other methods). Sinauer Associates, Sunderland, MA.

Thompson, J.D., Gibson, T.J., Plewniak, F., Jeanmougin, F., Higgins, D.G., 1997. The CLUSTAL_X windows interface: flexible strategies for multiple sequence alignment aided by quality analysis tools. Nuclei Acids Res. 25, 4876-4882.

Tsai, S.J., Huang, W.F., Wang, C.H., 2005. Complete sequence and gene organization of Nosema spodopterae rRNA gene. J. Eukaryot. Microbiol. 52, 52-54.

Zander, E., 1909. Tierische Parasiten als Krankenheitserreger bei der Biene. Leipziger Bienenzeitung 24, 147-150, 164-166. 